OPEN ACCESS

Edited by:

Bostjan Kobe,

The University of Queensland,

Australia

Reviewed by:

Tudor Moldoveanu,

St. Jude Children's Research Hospital,

United States

Sarah J. Poynter,

Wilfrid Laurier University, Canada

${ }^{*}$ Correspondence:

Pedro J. Esteves

pjesteves@cibio.up.pt

Specialty section:

This article was submitted to

Comparative Immunology,

a section of the journal

Frontiers in Immunology

Received: 26 July 2021

Accepted: 17 August 2021

Published: 01 September 2021

Citation:

Áqueda-Pinto A, Alves $L Q$,

Neves F, McFadden G, Jacobs BL,

Castro LFC, Rahman MM and

Esteves PJ (2021) Convergent

Loss of the Necroptosis Pathway in

Disparate Mammalian Lineages Shapes Viruses Countermeasures.

Front. Immunol. 12:747737.

doi: 10.3389/fimmu.2021.747737

\section{Convergent Loss of the Necroptosis Pathway in Disparate Mammalian Lineages Shapes Viruses Countermeasures}

\author{
Ana Águeda-Pinto ${ }^{1,2}$, Luís Q. Alves ${ }^{3}$, Fabiana Neves ${ }^{1}$, Grant McFadden ${ }^{4}$, \\ Bertram L. Jacobs ${ }^{4,5}$, L. Filipe C. Castro ${ }^{2,3}$, Masmudur M. Rahman ${ }^{4}$ \\ and Pedro J. Esteves ${ }^{1,2,6 *}$ \\ ${ }^{1}$ CIBIO/InBio-Centro de Investigação em Biodiversidade e Recursos Genéticos, Universidade do Porto, Vairão, Portugal, \\ 2 Departamento de Biologia, Faculdade de Ciências, Universidade do Porto, Porto, Portugal, ${ }^{3}$ CIIMAR/CIMAR, Centro \\ Interdisciplinar de Investigação Marinha e Ambiental, Universidade do Porto, Matosinhos, Portugal, ${ }^{4}$ Center for \\ Immunotherapy, Vaccines and Virotherapy, The Biodesign Institute, Arizona State University, Tempe, AZ, United States, \\ ${ }^{5}$ School of Life Sciences Center for Immunotherapy, Vaccines and Virotherapy, Biodesign Institute, Arizona State University, \\ Tempe, AZ, United States, ${ }^{6}$ CITS-Centro de Investigação em Tecnologias da Saúde, Instituto Politécnico de Saúde do \\ Norte (IPSN), Cooperativa de Ensino Superior Politécnico e Universitário (CESPU), Gandra, Portugal
}

Programmed cell death is a vital process in the life cycle of organisms. Necroptosis, an evolutionary form of programmed necrosis, contributes to the innate immune response by killing pathogen-infected cells. This virus-host interaction pathway is organized around two components: the receptor-interacting protein kinase 3 (RIPK3), which recruits and phosphorylates the mixed lineage kinase-like protein (MLKL), inducing cellular plasma membrane rupture and cell death. Critically, the presence of necroptotic inhibitors in viral genomes validates necroptosis as an important host defense mechanism. Here, we show, counterintuitively, that in different mammalian lineages, central components of necroptosis, such as RIPK3 and MLKL, are deleted or display inactivating mutations. Frameshifts or premature stop codons are observed in all the studied species of cetaceans and leporids. In carnivores' genomes, the MLKL gene is deleted, while in a small number of species from afrotheria and rodentia premature stop codons are observed in RIPK3 and/or MLKL. Interestingly, we also found a strong correlation between the disruption of necroptosis in leporids and cetaceans and the absence of the N-terminal domain of E3-like homologs (responsible for necroptosis inhibition) in their naturally infecting poxviruses. Overall, our study provides the first comprehensive picture of the molecular evolution of necroptosis in mammals. The loss of necroptosis multiple times during mammalian evolution highlights the importance of gene/pathway loss for species adaptation and suggests that necroptosis is not required for normal mammalian development. Moreover, this study highlights a co-evolutionary relationship between poxviruses and their hosts, emphasizing the role of host adaptation in shaping virus evolution.

Keywords: necroptosis, mammalian lineages, RIPK3, MLKL, convergent gene loss, host-range interactions 


\section{INTRODUCTION}

Sensing of viral pathogens by the host cells is critical for animal survival. Thus, a variety of molecular responses, including the induction of inflammatory cytokines, chemokines and interferons, as well as the activation of cell-death pathways that provide clearance of pathogen-infected cells, require sensing of pathogen associated molecular patterns for activation. Although apoptosis has long been considered a critical clearance mechanism to control viral spread, caspase-independent cell death, or programmed necrosis, has recently emerged as an alternative death pathway that dominates under specific conditions (1).

Necroptosis is an inflammatory form of regulated necrosis that acts as an alternative host defense pathway during some viral infections and plays a major role in the killing and removal of pathogen-infected cells (1-3). Activation of necroptosis follows an intracellular signaling cascade that is dependent on the receptor-interacting serine/threonine-protein kinase 3 (RIPK3) and its substrate, the mixed lineage kinase like protein (MLKL) downstream of death receptors (DRs) and pattern-recognition receptors (PPRs) (Figure 1) (4, 5). Several pathway-specific adaptor proteins that contain a RIP homotypic interaction motif (RHIM-domain) can activate RIPK3-induced necroptosis
(Figure 1). For example, when there is an interference or loss of function of caspase-8, the induction of necroptosis through the use of DRs results in the recruitment of RIPK1, which subsequently exposes its RHIM-domain to recruit RIPK3 (68). Apart from RIPK1, the TIR-domain-containing adaptorinducing IFN $\beta$ (TRIF), an essential protein downstream of Toll-like receptor (TLR)3/4 and the Z-DNA binding protein (ZBP1), also directly activate RIPK3 $(9,10)$ (Figure 1). Exposure of RIPK3 to a RHIM adaptor (RIPK1, TRIF or ZBP1) is a crucial step in the initiation of necroptosis, as these proteins activate the downstream executor of necroptosis, MLKL, that destabilizes the plasma membrane integrity leading to cell swelling followed by membrane rupture of infected cells and release of damageassociated molecular patterns (DAMPs) (3, 11). Thus, necroptosis provides a critical extra defense mechanism against pathogen infection, facilitating the elimination of virus-infected cells before the production of progeny virions. The importance of necroptosis for host defense is further supported by the identification of viral inhibitors of necroptosis, like is the case of Vaccinia virus (VACV) E3 protein and the murine cytomegalovirus (MCMV) M45 protein $(9,11)$.

Necroptosis has a major role in protecting cells against viral infection (1-3). However, despite the recent advances to understand the molecular regulation of this unique pathway, it

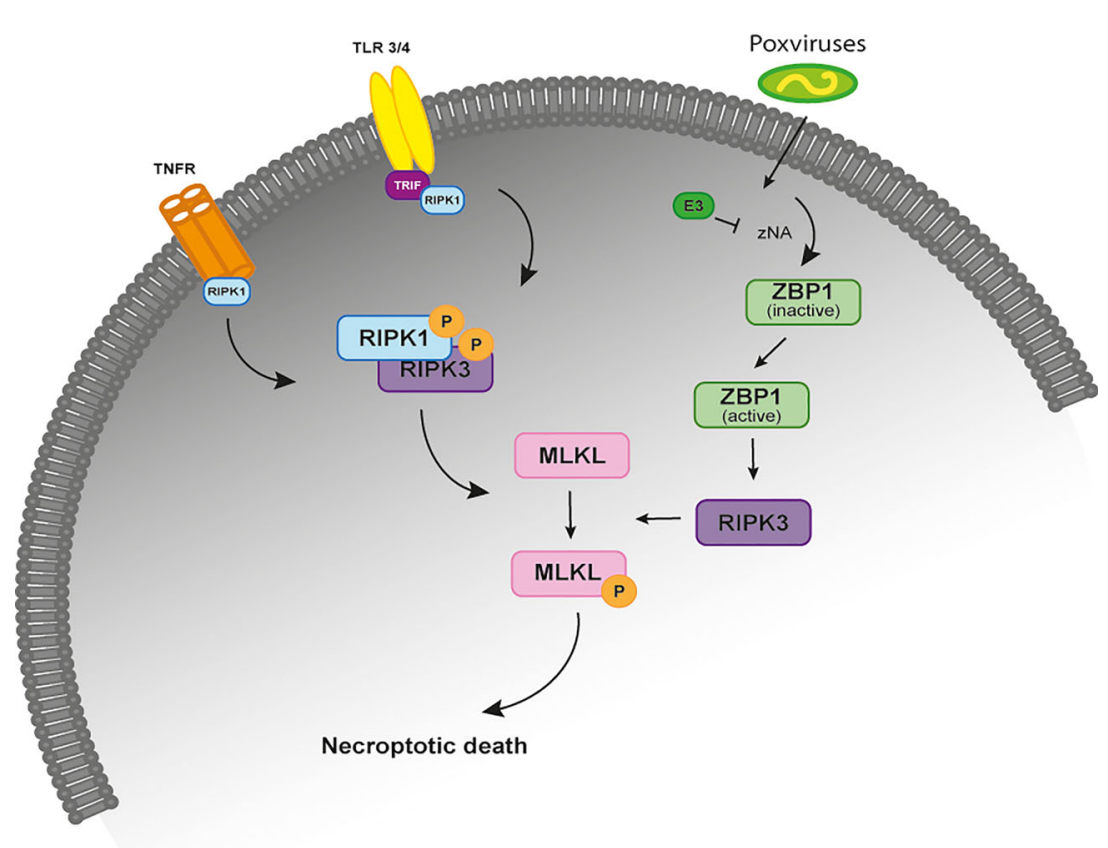

FIGURE 1 | The necroptosis signaling pathway. Simplified schematic representation of the necroptosis signaling pathway upon stimulation of the TNFR, TRF3/4 and infection by poxviruses. All of these necroptosis-inducing signals converge on the kinase RIPK3, which is activated through the homotypic interaction with RIPK1 or other RHIM-containing proteins, such as TRIF and DAI. When the activity of caspase-8 is inhibited, binding of TNF to TNFR1 leads to the phosphorylation and activation of RIPK1 that binds to RIPK3 through their RHIM domains to form a protein complex (necrosome). Activated RIPK3 recruits MLKL that oligomerizes and translocates to the plasma membrane to cause necroptosis. In TLR3- and TLR4-induced necroptosis, TRIF is required for the activation of RIPK3. ZBP1 is required for the activation of RIPK3 in response to the presence of Z-form nucleic acids. In VACV-infected cells, the poxviral E3 protein binds to VACV-induced Z-form nucleic acid, preventing RIPK3-induced necroptosis. TNFR, tumor necrosis factor receptor; TLR 3/4, toll-like receptor; TRIF, TIR-domain-containing adaptor-inducing IFN $\beta$; RIP, receptor-interacting protein kinase; ZBP1, Z-DNA binding protein; MLKL, mixed-lineage kinase domain like. 
is still unclear whether the necroptotic cell death pathway acts as a universal cell death program in mammals. Previous studies suggested that components of necroptosis are absent in the genomes of extant birds and marsupials. Interestingly, within the Mammalia class, it was previously reported that order Carnivora lost the MLKL gene (12). Taken together these reports suggest some degree of plasticity in the conservation of necroptosis responses. Here, we address the molecular evolution of the necroptotic pathway in multiple mammalian lineages. We show that during mammalian evolution, necroptosis was convergently inactivated several times in mammalian lineages. Remarkably, we also report that mammalian orders that lost the necroptotic pathway display infection episodes by poxviruses that have lost the ability to inhibit this pathway, showing a coevolutionary relationship between host adaptation in shaping virus evolution.

\section{METHODS}

\section{Genomic Approach to Detect Genes Associated With the Necroptosis Pathway}

To detect intact and inactivated genes, we first identified the key genes of the necroptotic pathway (i.e., RIPK1, RIPK3 and MLKL) in the human (H. sapiens) and mouse (Mus musculus) reference genomes and looked for the presence of orthologues in existing genome sequence databases from 67 different species that belong to the 9 main mammalian orders/superorders: primates, rodents, lagomorpha, chiroptera, carnivora, perissodactyla, artiodactyla, cetacea, and afrotheria (Supplementary File 1). We did not only search for the complete loss of exons or entire genes, but also searched for insertions and deletions that shift the reading frame, frame-preserving insertions that create a premature stop codon and substitutions that create an in-frame stop codon. The respective search methodology had been previously applied to the identification of different homologues of different annotated mammalian genomes $(13,14)$. To further ensure that all gene loss events discussed in this study are real and not due to sequencing errors, we validated them either by sequencing of samples or by using a curated bioinformatic pipeline (see below).

\section{Amplification and Sequencing of RIPK1 and RIPK3 Nucleic Acid Sequences From Lagomorpha Species}

In contrast to the majority of mammalian orders, lagomorpha only presents three annotated genomes: the European rabbit (Oryctolagus cuniculus, accession \# GCA_000003625.1), the American Pika genome (Ochotona princeps, accession \# GCA_014633375.1) and the plateau pika (O. curzoniae, accession \# GCA_017591425.1). Given the importance of lagomorphs for this study, samples from different lagomorpha species were used to obtain the nucleic coding sequence from RIPK1. For that, RNA was extracted from tissues of O. cuniculus cuniculus, O. cuniculus algirus, Lepus americanus, L. europaeus, L. timidus, L. granatensis, Sylvilagus floridanus, S. bachmanis, O. princeps and O. collaris samples, using the Qiagen DNeasy Blood
\& Tissue kit (Qiagen, USA) following manufacturer's instructions. Synthesis of cDNA was achieved by using SuperScript III Reverse Transcriptase (Invitrogen, USA). Primers were designed according to the RIPK1 transcript from O. cuniculus [Accession \# XM_017350509.1] (Forward 5'ATGTCTTTGGATGACATTAAAATG-3' and Reverse 5'CTACTTCTGGCTGAGCTGTATC-3') and used to amplify the samples mentioned before. Phusion ${ }^{\circledR}$ High-Fidelity DNA Polymerase (Finnzymes, Espoo, Finland) was used in the PCR amplification and the conditions included an initial denaturation $\left(98^{\circ} \mathrm{C}\right.$ for $\left.3 \mathrm{~min}\right), 35$ cycles of denaturation $\left(98^{\circ} \mathrm{C}\right.$ for $\left.30 \mathrm{~s}\right)$, annealing $\left(60^{\circ} \mathrm{C}\right.$ for $\left.15 \mathrm{~s}\right)$ and extension $\left(72^{\circ} \mathrm{C}\right.$ for $\left.30 \mathrm{~s}\right)$ followed a final extension $\left(72^{\circ} \mathrm{C}\right.$ for $\left.5 \mathrm{~min}\right)$.

From our initial search, the RIPK3 gene was not annotated in the European rabbit. However, after mapping the location of RIPK3 based on its location in H. sapiens, M. musculus and $O$. curzoniae, we were able to identify a partial RIPK3 sequence in the European rabbit genome that presented an early stop codon. To exclude potential artifacts that can mimic real geneinactivating mutations, a forward (5'-ATGTCTTCTGTCAAAT TGTGG-3') and a reverse (5'-ACTGCCTGCATCAGGATC-3') primer were designed based on the parcial RIPK3 sequence and were used to amplify the same region in the genomes from $O$. cuniculus cuniculus, S. floridanus, L. americanus, L. europaeus and L. saxatilis. For that, genomic DNA was extracted using the Qiagen DNeasy Blood \& Tissue kit (Qiagen, USA) according to the manufacturer's instructions. Phusion ${ }^{\circledR}$ High-Fidelity DNA Polymerase (Finnzymes, Espoo, Finland) was used in the PCR amplification and the conditions included an initial denaturation $\left(98^{\circ} \mathrm{C}\right.$ for $\left.3 \mathrm{~min}\right), 9$ cycles of denaturation $\left(98^{\circ} \mathrm{C}\right.$ for $\left.30 \mathrm{~s}\right)$, annealing $\left(66^{\circ} \mathrm{C}\right.$ for $\left.15 \mathrm{~s}\right)$ and extension $\left(72^{\circ} \mathrm{C}\right.$ for $\left.30 \mathrm{~s}\right)$ followed by more 25 cycles of denaturation $\left(98^{\circ} \mathrm{C}\right.$ for $\left.30 \mathrm{~s}\right)$, annealing $\left(61^{\circ} \mathrm{C}\right.$ for $\left.15 \mathrm{~s}\right)$ and extension $\left(72^{\circ} \mathrm{C}\right.$ for $\left.30 \mathrm{~s}\right)$ and a final extension $\left(72^{\circ} \mathrm{C}\right.$ for $\left.5 \mathrm{~min}\right)$.

Amplicons sequencing from RIPK1 and RIPK3 was performed with the ABI PRISM BigDye Terminator v3.1 Cycle Sequencing Kit and according to manufacturer's protocol; reactions were cleaned with Sephadex ${ }^{\mathrm{TM}}$ (GE Healthcare Life Sciences, UK) and applied on an ABI PRISM 310 Genetic Analyser (Life Technologies, Applied Biosystems, Carlsbad, CA, USA). The obtained RIPK1 coding sequences and the partial RIPK3 sequences from the different Lagomorphs have been deposited in the GenBank database under the accession numbers that are shown in Supplementary File 2. All samples were supplied by CIBIO/InBIO, Vairão, Portugal and used in previous studies $(15,16)$. No animals were captured, handled, or killed specifically for the purpose of this study.

\section{Detailed Analysis on the Cetacean Genomes}

Briefly, NCBI gene annotations for the gene orthologues of MLKL and RIPK3 were initially screened via PseudoChecker (pseudochecker.ciimar.up.pt), which evaluates the coding condition of a gene $(17,18)$. For each gene, a PseudoChecker analysis was run (default parameters), using the Bos taurus (cow) gene orthologue as a comparative input (NCBI Accession ID 
regarding cow MLKL: XM_002694707.6; RIPK3: XM_024997365.1), as well as the genomic sequences encompassing the putative ORF of the orthologous counterpart of each target species, directly exported from the NCBI genome browser. Through PseudoIndex, a built-in assistant metric, we quickly assessed the erosion status of the tested genes on a discrete scale ranging from 0 (coding) to 5 (pseudogenized) (17). Subsequent manual annotation was performed by importing the previously collected genomic sequences into Geneious Prime 2020 software (www.geneious.com) (19) and determining each gene's CDS using as reference cow's MLKL and RIPK3 orthologues sequences. In detail, per gene and species, using the built-in map to reference tool (highest sensitivity parameter selected), each (3' and 5' untranslated region-flanked) reference coding-exon was mapped against each target genomic sequence. Exons alignments were further screened for gene disruptive mutations, including in-frame premature stop codons, frameshift, and splice site mutations (any deviation from the consensus donor splice site GT/GC or the consensus acceptor splice site $A G)$.

To inspect if the identified genetic lesions were not rendered as result of sequencing and/or genome assembly artifacts, we performed mutational validation (one per gene and species), resorting of raw genomic sequencing reads, retrieved from two independent genomic projects from the NCBI sequence read archive (SRA), when available. Explicitly, blastn searches were directed to the selected SRA projects, using the nucleotide sequence portion containing the selected mutation(s) as a query. The matching sequencing reads were downloaded into Geneious Prime 2020 (19) software and mapped against the manually annotated mutation (highest sensibility parameter selected), confirming, or not, the presence of the identified mutation.

\section{Phylogenetic and Molecular Evolutionary Analyses}

The complete dataset of RIPK1, RIPK3 and MLKL proteins was aligned in BioEdit Sequence Alignment Editor using Clustal W (20), followed by manual corrections when necessary. Amino acid alignments were then used to infer Maximum Likelihood (ML) phylogenetic trees using MEGA $X$ (21), with the substitution models $\mathrm{JTT}+\mathrm{G}+\mathrm{F}, \mathrm{JTT}+\mathrm{G}$ and $\mathrm{HKY}+\mathrm{G}+\mathrm{I}$, respectively; determined using ProtTest (22).

Given the fact that RIPK3 and MLKL proteins are highly divergent across the studied mammalian species, we decided not to perform any evolutionary analysis using these alignments. To look for signatures of natural selection operating in the RIPK1 alignment, we used HyPhy software implemented in the Datamonkey Web server (23), to detect codons under selection: the Single Likelihood Ancestor Counting (SLAC) model, the Fixed Effect Likelihood (FEL) method (24), the Random Effect Likelihood, the Mixed Effects Model of Evolution (MEME) (25) and Fast Unbiased Bayesian AppRoximation (FUBAR) (26) methods were used. To avoid a high false positive rate, codons with p-values $<0.05$ for SLAC, FEL and MEME models and a posterior probability $>0.95$ for
FUBAR were accepted as candidates for selection. For a more conservative approach, only residues identified as being under positive selection in three or more ML methods were considered.

\section{Analysis of VACV E3 Homologues}

VACV E3 homologues encoded by different poxviruses $(=11)$ were retrieved from the NCBI database (https://www.ncbi.nlm. nih.gov/) and aligned in the BioEdit Sequence Alignment Editor using Clustal W (20), followed by manual corrections when necessary. Amino acid alignments of the representative E3-like proteins were used to generate schematic diagrams using the COBALT program from the NCBI database.

\section{RESULTS}

Necroptosis has a significant role in protecting cells against viral infection (1-3). However, despite recent advances to understand the molecular regulation of this unique pathway, it is still unclear whether the necroptotic cell death pathway acts as a universal cell death program in mammals. Here, taking advantage of genomic collection databases and the use of Leporid samples (see Methods section for more information), the goal is to better understand the molecular evolution of the necroptotic pathway in different mammalian lineages.

\section{RIPK1 Protein Is Under Evolutionary Conservation in Mammals}

Human RIPK1 is a multidomain protein composed of an Nterminal Ser/Thr kinase, a C-terminal death binding domain that mediates binding to DRs and an intermediate domain that includes a K377 ubiquitination site and an RHIM motif that binds to other RHIM-containing proteins (27). Due to the importance of RIPK1 as an adaptor molecule, previous phylogenetic analysis suggested that RIPK1 probably emerged in the ancestor of vertebrates (12). In accordance, our search detected the presence of RIPK1 homologues in all the studied mammalian lineages (Figure 2A and Supplementary Files 1-3).

To look for evidence of potential selection pressures acting on the different domains of RIPK1 protein, we used the dataset of mammalian sequences mentioned above and implemented an ML approach, by using the Datamonkey software (see Methods section for more information). For most protein-coding genes, the rate between nonsynonymous and synonymous substitutions $(\mathrm{dN} / \mathrm{dS})$ is a measure of natural selection, with positive selection $(\mathrm{dN} / \mathrm{dS}>1)$ acting against the common genotype (28). In this study, we deduced ten sites that reflect strong positive selection pressure in RIPK1, while more than 200 amino acids were under negative selection (Figure 2B and Supplementary File 4). As seen in Figure 2B, residues identified as being under positive selection fall within or very close to the kinase domain (4 residues), the RHIM domain (5 residues) and the death domain (1 residue) of RIPK1 (residues under positive selection are marked as red circles). The $\mathrm{N}$-terminal kinase domain is known to present several essential residues for phosphorylation and ubiquitination (Ser14/15, 20, 161 and 166 and Lys115 and 


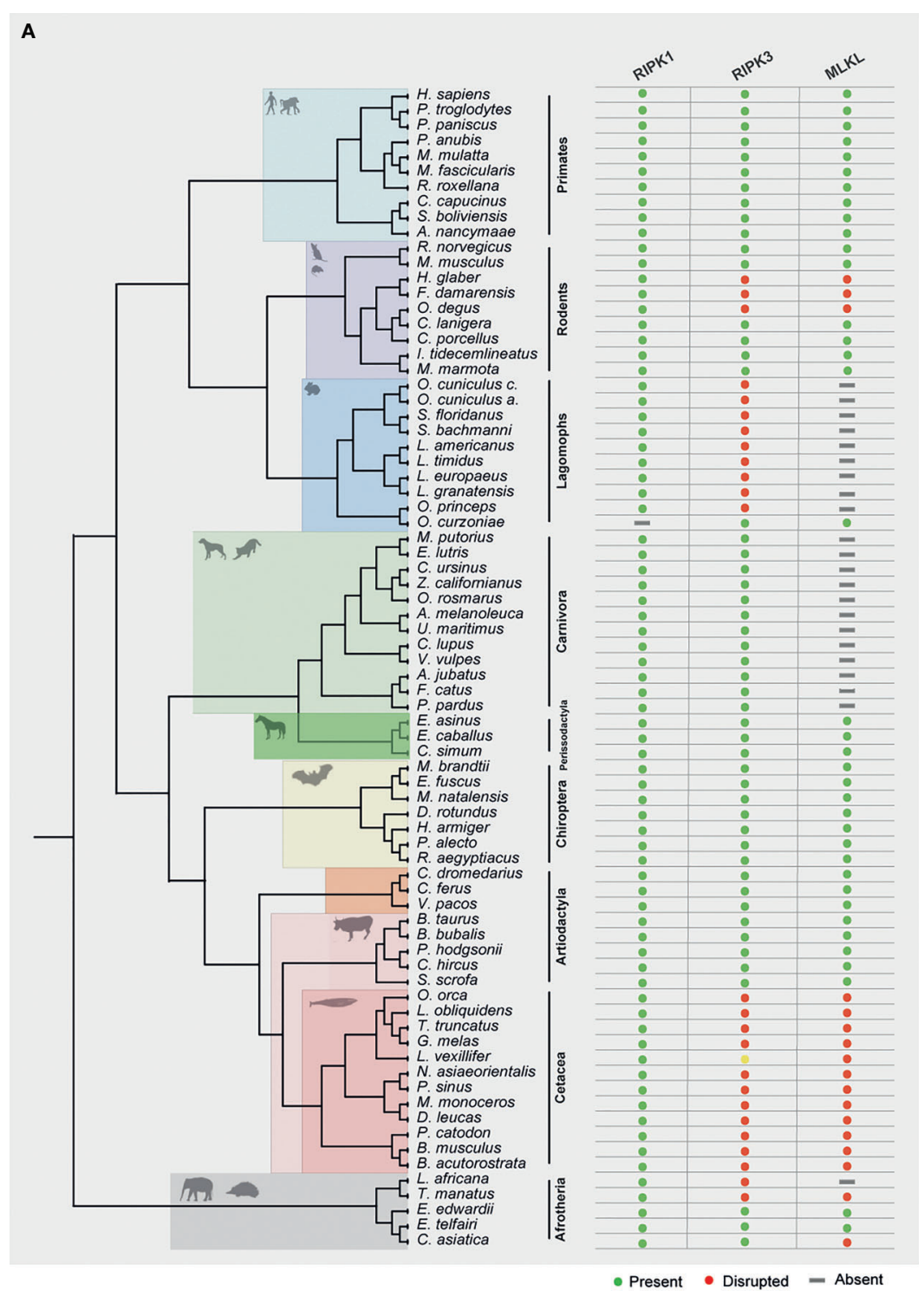

B

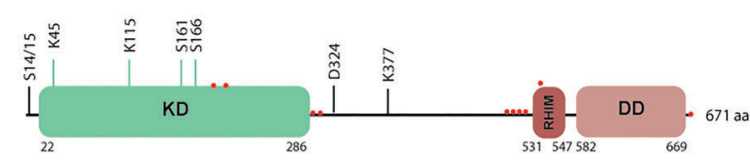

RIPK3

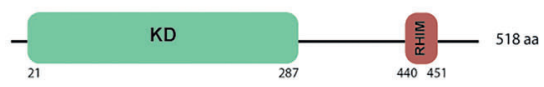

MLKL

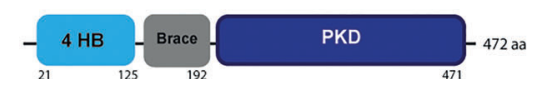

FIGURE 2 | Evolution of RIPK1, RIPK3 and MLKL in different mammalian lineages. (A) Phylogenetic tree showing the independent lineages that lost necroptotic core proteins (RIPK3 and MLKL) during evolution. Green circles represent genes that are present in the studied species, red circles represent genes that are disrupted, yellow circles represent genes that have incomplete assemblies and grey rectangles indicate that genes were not found in those species genomes.

(B) A schematic diagram of RIPK1, RIPK3 and MLKL domains. RIPK1 contains an N-terminal kinase domain (KD), an intermediate domain with a RIP homotypic interaction motif (RHIM), and a C-terminal death domain (DD). The phosphorylation and ubiquitination sites are indicated above the RIPK1 domains. Red circles represent residues that are under positive selection. RIPK3 contains a KD and a RHIM domain. MLKL is composed of an N-terminal bundle four-helix bundle (4HD) domain that is regulated by the $\mathrm{C}$-terminal pseudokinase domain (PKD). 
163), regulation of necroptosis and RIPK1-dependent apoptosis (29). Interestingly, the codons under positive selection fall only at the end of the N-terminal domain. A considerable portion of the negatively selected sites fall in the critical regions for phosphorylation and ubiquitination (Supplementary File 4), suggesting that the beginning of the RIPK1 protein is under strong purifying selection. The same was also observed for the rapidly evolving sites of the RHIM domain, which were grouped only at the beginning of this domain (Figure 2B). It was previously shown that the RHIM domain has a crucial conserved core motif of 12 amino acids that resides at the end of this domain $(30,31)$. Indeed, changing four consecutive amino acids to alanine within this core region abrogates interaction between RHIM domains and, as a consequence, necroptosis (30, 31). Our findings that the positively selected residues did not overlap with the core motif of 12 amino acids further support the importance of the conservation of this region. Interestingly, in the RIPK1 death domain that is known to mediate homodimerization as well as heterodimerization with other DD-containing proteins, such as FADD, TNFR1 and Fas (29, 32), no positively selected sites were found, with most of the domain being under negative pressure (Supplementary File 4). Collectively, our results show signatures of positive selection occurring at the end of the N-terminal kinase domain and at the beginning of the RHIM domain of RIPK1 proteins. Interestingly, these residues do not overlap with domains known to be fundamental for RIPK1-dependent apoptosis and necroptosis, suggesting that these domains might be under evolutionary conservation and possibly functional constraint for the studied mammals.

\section{Convergent Erosion of RIPK3 and MLKL in Mammalian Lineages}

RIPK3 and MLKL form the core of the necroptotic machinery and both are, as a consequence, important for necroptosis induction in mice and humans downstream of PRRs and DRs $(1,4,5)$. To further understand the evolutionary history of the necroptotic pathway in mammals, we performed detailed sequence and phylogenetic analyses for RIPK3 and MLKL homologous proteins (Supplementary File 3). Our screens for RIPK3 and MLKL genes revealed evidence of pseudogenization in five mammalian lineages: order rodentia, lagomorpha, carnivora, cetacean and in the superorder afrotheria (Figure 2A). Given the fact that $M L K L$ pseudogenes have been previously identified in carnivores, they will not be discussed in detail here (12).

\section{Variation of the Necroptotic Pathway Within Afrotherian and Rodent Families}

In afrotherian and rodent lineages, we found that the necroptotic pathway was missing in some species (Figure 2A). In rodents, two species from the Bathyergidae family and one species from the Octodontidae family presented early stop codons in both RIPK3 and MLKL, resulting in the disruption of necroptosis. In the naked mole-rat (Heterocephalus glaber), RIPK3 presented a premature stop codon in exon 6 resulting in a shorter version of this protein (Supplementary File 5). In the common degu (Octodon degus) and in the damaraland mole-rat (Fukomys damarensis), both RIPK3 and MLKL proteins presented several premature stop codons (Supplementary File 5). However, disruption of these proteins appears to have occurred in an independent way, rather than in a common ancestral. Given the fact that RIPK3 and MLKL present signs of pseudogenization, it is expected that in the naked mole-rat, common degu and damaraland mole-rat necroptosis is disrupted. Our studies also revealed that species from Afrotherian families, including the African bush elephant (Loxodonta africana, family Elephantidae) and the Cape golden mole (Chrysochloris asiatica, family Chrysochloridae) presented early stop codons in RIPK3 and MLKL, respectively, while the West Indian manatee (Trichechus manatus, family Trichechidae) present early stop codons in both genes (Supplementary File 5). However, our results also show that the Cape elephant (Elephantulus edwardii, family Macroscelididae) and the lesser hedgehog tenrec (Echinops telfairi, family Tenrecidae) present intact copies of the RIPK3 and $M L K L$ genes, indicating that RIPK 3 and MLKL were present in early stages of Afrotheria evolution, but must have been lost later in specific lineages, resulting in the existence of alternative modes of necroptosis inactivation.

\section{The Necroptotic Pathway Is Disrupted in Lagomorphs}

The Order Lagomorpha is divided into two families, Ochotonidae and Leporidae, which diverged around 30-35 Mya (33). While Ochotonidae is only composed of one extant genus, Ochotona, the Leporidae family includes 11 genera, including Lepus, Sylvilagus and Oryctolagus (34). Using the methods described previously, we were only able to identify RIPK3 and MLKL transcripts for plateau Pika (O. curzoniae), while no RIPK3 and $M L K L$ transcripts were found for the European rabbit (O. cuniculus). For the American Pika (O. princeps), incomplete genome assemblies in the vicinity of the $R I P K 3$ and $M L K L$ regions made retrieving the sequence of these genes impossible. By evaluating RIPK3 gene from human and mouse and its genomic context, we were able derive a partial RIPK3 nucleotide sequence from the European rabbit genome, which displays a frameshift mutation caused by the insertion of a single nucleotide. It is well known that accurately detecting geneinactivation mutations in these alignments poses a number of challenges like, for example, sequencing errors and cases of assembly incompleteness. For this reason, we assessed the accuracy of our database prediction by sequencing that same genomic region in different Leporid species, representative of different genera (Lepus, Sylvilagus and Oryctolagus). From the obtained results, we confirmed the insertion of 1 nucleotide $(+\mathrm{G}$, exon 3) not only in the European rabbit RIPK3, but also in species from genus Lepus and Sylvilagus, suggesting that disruption of RIPK3 gene occurred in a common ancestral and was maintained throughout Leporid evolution (Figure 3A). During necroptosis, activated RIPK3 phosphorylates and activates MLKL, which results in its recruitment and oligomerization in the plasma membrane leading to rupture 
A

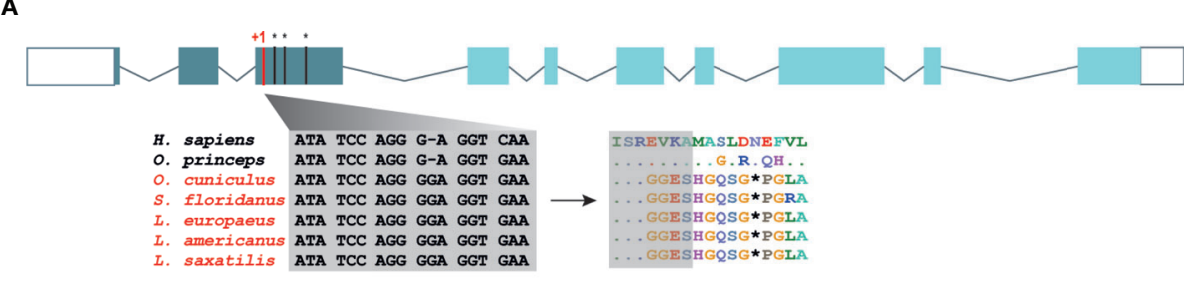

B

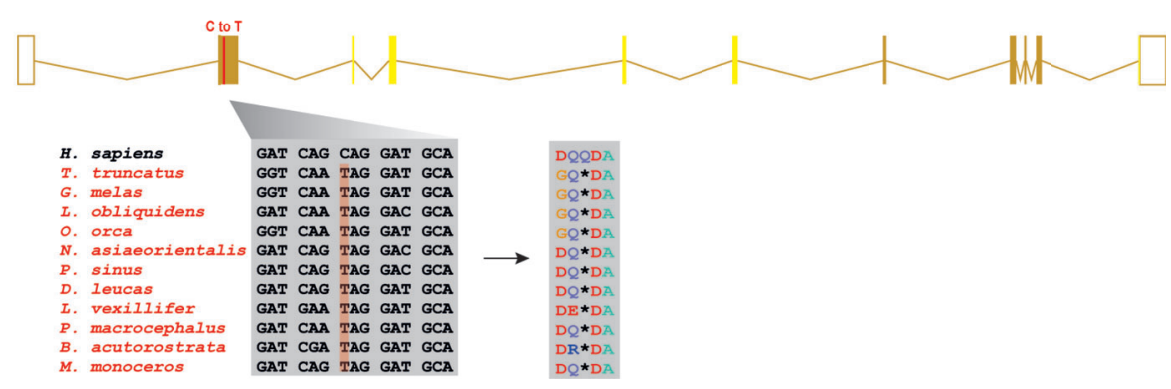

FIGURE 3 | Loss of RIPK3 and MLKL genes in the steam lineage of Leporids and Cetaceans. (A) Genomic analysis of the first tree exons from Leporids. In Leporids, RIPK3 was lost as a result of a shared insertion $(+G)$ in the third exon that resulted in the appearance of several premature stop codons. (B) A point mutation (C to T) in all the studied cetacean species indicates that MLKL inactivation occurred in Cetacea steam lineage. Moreover, 9 out of the 11 studied species (excluding B. acutorostrata and M. Monoceros) lost exon 2, 3, 4 and 5 throughout evolution (represented by faint yellow). Premature stop codons are represented by an asterisk (*).

and cell death $(1,4)$. Interestingly, and despite all of our efforts, no $M L K L$ transcripts or MLKL protein accumulation were found in any of the studied Lagomorphs (data not shown). Detailed analysis of the upstream and downstream MLKL flanking genes in both human and mouse genomes reveal that MLKL resides between the ring finger and WD repeat domain 3 protein (RFWD3) and fatty acid 2-hydroxylase (FA2H) genes (Figure 4). Accordingly, although there are no gaps or incomplete genomic assemblies surrounding that region in the European rabbit genome, we were not able to retrieve a complete or partial MLKL gene, suggesting once more that this gene is not present in these mammals. Together, our results suggest that the studied leporid species are deficient in the core proteins of the necroptotic pathway, and that RIPK3 inactivation occurred at the stem Leporid branch and was maintained during evolution.

\section{Inactivation of Necroptosis Components in Cetacea}

Modern Cetacea comprises Mysticete (or baleen whales) and Odontocete (or toothed whales) and are the most specialized and diversified group of mammals (35). Comparative analysis of cetacean genomes has already provided important insights into the unique cetacean traits and aquatic specializations $(13,36,37)$. For our screen, Odontocetes were represented by 12 species belonging to five different families (Delphinidae, Phocoenidae, Monodontidae, Lipotidae and Physeteridae), and Mysticetes were represented by the common minke whale from Balaenopteridae family. In Cetacean species, the disruption of RIPK3 occurred at different positions depending on the studied species: a frameshift mutation was identified in exon 6 of two
Delphinidae species, as well as in exon 8 of two Phocoenidae species, one Monodontidae species and one Balaenopteridae species. There was also evidence of two species (one species from Monodontidae and another from Lipotidae families) with RIPK3 pseudogenes based on the presence of a stop codon located in exon 2 (Supplementary File 6). Interestingly, while Cetacean RIPK3 inactivation appears to be a result of different mutations depending on the studied species, our results show a shared mutation in the exon 1 of MLKL in all cetacean species (Figure 3B). Moreover, this premature stop codon leads to the absence of exon 2, 3, 4 and 5 in most cetacean species, which very likely results in this gene inactivation. Given the presence of an inactivating mutation that is shared between mysticetes and odontocetes, the most parsimonious hypothesis suggests that they occurred before the split of these two clades in the common ancestral branch of Cetacea.

\section{Diversity Among the Poxvirus Encoded E3-Like Necroptosis Antagonists}

Previously, it was shown that the N-terminus of VACV E3 competes with ZBP1 for binding to virus-induced Z-nucleic acid, being a key component to inhibit the action of IFN and induction of necroptosis (38) (Figure 1). E3-like encoded proteins are composed of a carboxy (C)-terminal doublestranded RNA binding domain (dsRNA-BD) and an amino (N)-terminal Z-nucleic acid-binding domain (zNA-BD) (39, 40) (Figure 5A). Given the importance of the N-terminus region from VACV E3 protein against virus infection, we hypothesize that poxviruses that lack this region in their E3 homologs can still successfully replicate in their natural host 


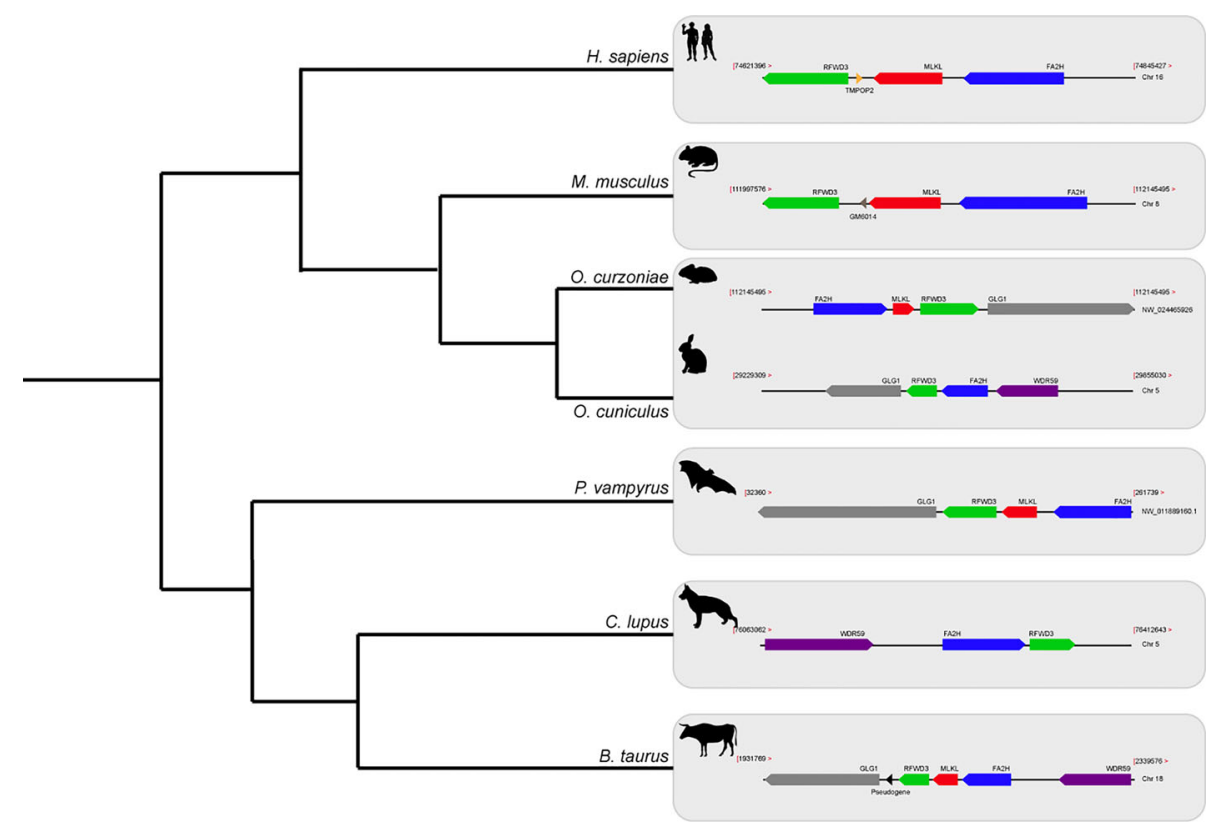

FIGURE 4 | Gene synteny of the genome regions containing MLKL gene in different mammals. Genomic regions containing the MLKL gene or its flanking genes in H. sapiens, M. musculus, O. curzoniae, O. cuniculus, P. vampyrus, C. lupus and B. taurus. Horizontal lines indicate chromosome fragments and coloured arrows identify genes and their orientation in the genome. Orthologous genes are indicated in the same colour and their names are indicated above/below. Black arrows indicate the presence of pseudogenes. RFWD3, ring finger and WD repeat domain 3; MLKL, mixed-lineage kinase domain like; FA2H, fatty acid 2-hydroxylase; GLG1, golgi glycoprotein 1; WDR59, WD repeat domain 59; TMPOP2, thymopoietin pseudogene; GM6014, ubiquitin-40S ribosomal protein S27a pseudogene; LOC788457, translationally-controlled 1 pseudogene.

because of a compromised necroptotic pathway. Among the E3L proteins, E3 from VACV is the best studied protein. However, E3 homologs can be found in orthopoxviruses, clade II poxviruses and parapoxviruses $(39,40)$. Recently, the genome characterization of CePV-TA identified two novel E3L homologs: CePV-TA-20 and CePV-TA-21 (41).

Our analysis on 11 different E3 homologues revealed that these are highly divergent: while CPXV 069 (Cowpoxvirus E3 homolog) and TATV 060 (Tateropoxvirus E3 homolog) presented identities of $>90 \%$ to VACV E3, E3L homologs from poxviruses like the Deerpoxvirus, Sheeppoxvirus and Yaba monkey poxvirus presented less than $40 \%$ identity (Figure 5B). Analysis of the two newly identified E3 homologs from CePV-TA shows that both present low identity to VACV E3, with CePV-TA-20 and CePV-TA-21 proteins only presenting sequence identity of $37 \%$ and $34 \%$, respectively (Figure 5B). It is known that at the amino acid level, the Cterminal of E3-like proteins display a higher level of sequence similarity than the N-terminal domain (42). Accordingly, the dsRNA-BD domain from CePV-TA-20 and CePV-TA-21 proteins also display a higher level of sequence similarity compared to other E3 homologs (Figure 5C), suggesting that in CeTV this domain might also target conserved antiviral dsRNA-activated pathways. Similar to what is observed for Monkeypox virus (MPXV) and Myxoma virus (MYXV) E3 homologues, both E3 homologs from CePV-TA present incomplete or disrupted $\mathrm{N}$-terminal zNA-BDs. As shown in
Figure 5C, CePV-TA-20 is missing 20 amino acids in its Nterminal domain. However, this region still retains the conserved LY and PPXW motifs, as well the basic KKCINR motif (Figure 5C) that are known to bind with Z-NA (40). Interestingly, MPXV F3 protein, lacking 37 amino acids from the $\mathrm{N}$-terminal domain, is not able to compete with ZBP1 and inhibit sensing (unpublished data), even though it retains the key residues important for binding to Z-NA (Figure 5C). While CePV-TA-20 and F3 proteins contain an incomplete zNA-BD, M029 and CePV-TA-21 proteins are missing most of their Nterminal zNA-BD (Figures 5B, C), suggesting a total inactivation of this domain and a loss of function regarding the inhibition of ZBP1-dependent necroptosis. Overall, our results show that the novel CePV-TA presents two E3L homologues that, like E3L homologues from MPXV and MYXV, present incomplete or disrupted N-terminal zNA-BD. The presence of an incomplete or disrupted $\mathrm{zNA}-\mathrm{BD}$ in E3L homologues highly suggests that these proteins cannot fully compete with ZBP1 to inhibit necroptosis induction. However, further studies will be necessary to fully comprehend the action of these proteins regarding complete necroptosis inhibition.

\section{DISCUSSION}

Necroptosis is an inflammatory form of cell death that is mediated by RIPK3 and MLKL and provides an extra defense 


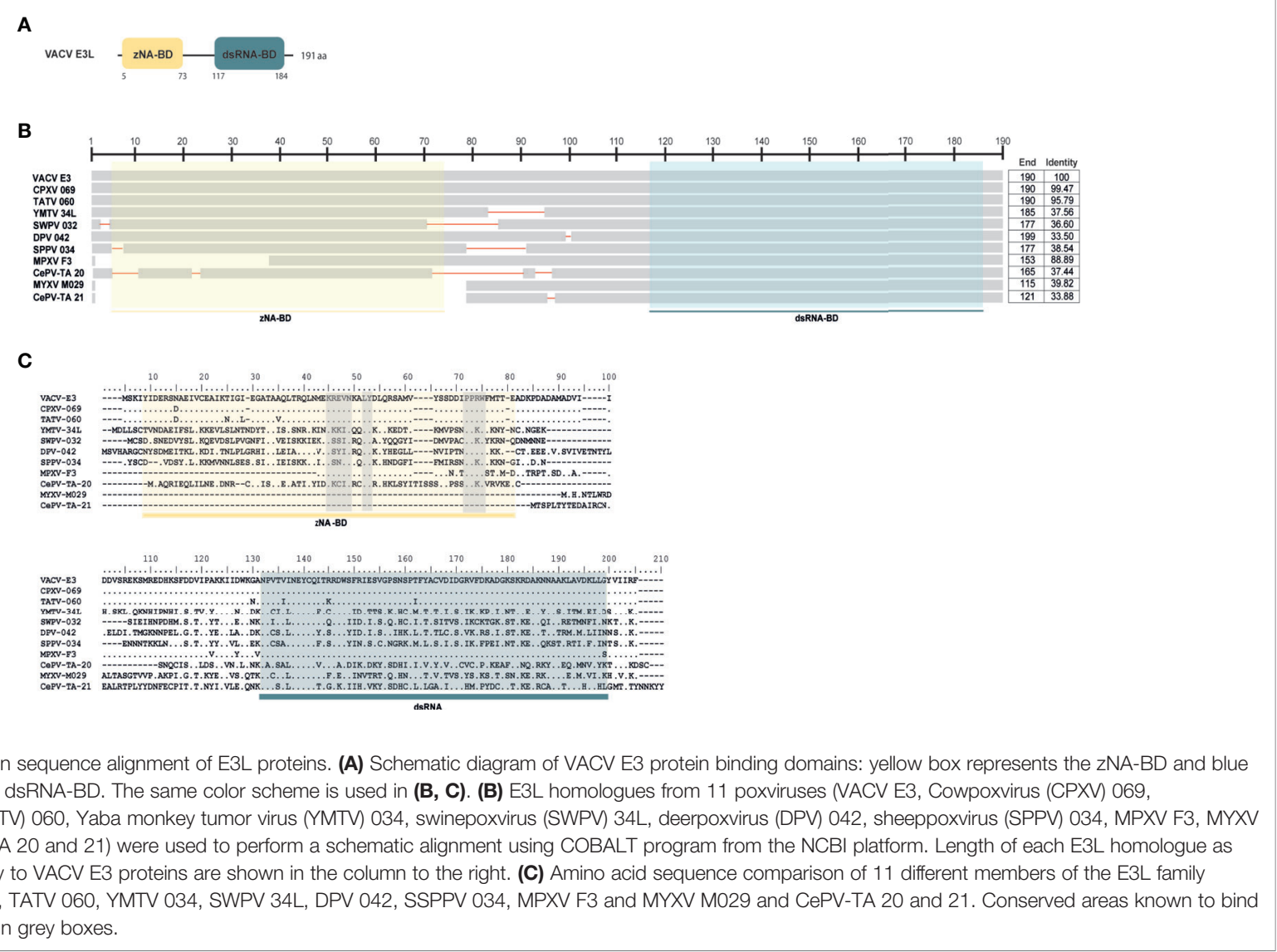

FIGURE 5 | Protein sequence alignment of E3L proteins. (A) Schematic diagram of VACV E3 protein binding domains: yellow box represents the zNA-BD and blue box represents the dsRNA-BD. The same color scheme is used in (B, C). (B) E3L homologues from 11 poxviruses (VACV E3, Cowpoxvirus (CPXV) 069, Tateropoxvirus (TATV) 060, Yaba monkey tumor virus (YMTV) 034, swinepoxvirus (SWPV) 34L, deerpoxvirus (DPV) 042, sheeppoxvirus (SPPV) 034, MPXV F3, MYXV M029 and CePV-TA 20 and 21) were used to perform a schematic alignment using COBALT program from the NCBI platform. Length of each E3L homologue as well as their identity to VACV E3 proteins are shown in the column to the right. (C) Amino acid sequence comparison of 11 different members of the E3L family including VACV E3, TATV 060, YMTV 034, SWPV 34L, DPV 042, SSPPV 034, MPXV F3 and MYXV M029 and CePV-TA 20 and 21. Conserved areas known to bind to zNA are shown in grey boxes.

mechanism against pathogen infection, facilitating the elimination of virus-infected cells before the production of progeny virions $(1-3)$. Given the crucial role of necroptosis in the innate immune response of humans and mice $(43,44)$, it was broadly accepted that this pathway was ubiquitous in mammals. Our results from 67 species across nine mammalian lineages provides the first comprehensive picture of the molecular evolution of necroptosis in mammals. We show that while RIPK1 is under evolutionary conservation, RIPK3 and MLKL are poorly conserved in lineages that evolved separately over the course of evolution.

A detailed analysis of RIPK3 and MLKL in mammals reveals a complex pattern where lagomorphs, cetaceans, carnivores and species from rodent and afrotheria lineages separately lost key components of the necroptotic pathway (Figure 2A). The order lagomorpha includes two big families, Ochotonidae and Leporidae (33). The presence of the same frameshift mutation in Leporid species $(+G$, Figure 3A) suggests that the disruption of the necroptotic pathway occurred early, but only after the bifurcation between Ochotona and Leporid given that the plateau Pika presents intact RIPK3 and MLKL proteins (Figure 2A). Despite all efforts, and despite the complete genome assembly surrounding the MLKL flanking genes in the European rabbit genome, no partial or complete MLKL gene was found, indicating that this gene is deleted in the European rabbit genome and possibly in the remaining Leporid species
(Figure 4). The core genes of the necroptotic pathway also presented premature stop codons in cetaceans. In the studied cetaceans, the MLKL gene presented a common stop codon in the first exon, resulting in the inactivation of this gene (Figure 3B). Again, the presence of similar patterns of pseudogenization in RIPK3 or MLKL genes within species of the same order infer that disruption of these genes occurred before their diversification and was maintained throughout evolution. On the other hand, RIPK3 disruption in cetaceans appears to be the result of insertions or deletions that are not shared between closely related species, but rather specific to each species (Supplementary File 6), suggesting that these disrupting mutations occurred later in evolution when compared to MLKL. The addition of some rodents as well as afrotheria species to the list of mammals that have disrupted necroptotic pathways, raises the possibility that other closely related species might have lost this pathway after the diversification of these lineages. It is currently believed that activation of the RIPK 3 and recruitment of MLKL are critical steps during necroptosis. For example, deleting either RIPK3 or MLKL can lead to the suppression of skin and liver inflammation in mice $(45,46)$. Moreover, when mice are treated intravenously with a highdoses of TNF, there appears to be no differences between RIPK3deficient and MLKL-deficient mice (5), substantiating the premise that MLKL follows RIPK3 in the necroptotic signalling. However, this appears not to be the case for all 
necroptotic cell death responses, as different studies revealed alternative pathways for MLKL and RIPK3-dependent programmed necrosis that are executed in the absence of RIPK3 or MLKL, respectively $(47,48)$. For example, Inositol Phosphate metabolites have been previously shown to be required for human MLKL activation by directly acting on the $\mathrm{N}$-terminal $4 \mathrm{HB}$ to dislodge an inhibitory region, revealing that MLKL-mediated necroptosis can also be controlled by a metabolite (49). To date, there are no studies suggesting that RIPK3/MLKL double-knockout mice are still able to induce necroptosis, which indicates that species that have disrupted RIPK3 and MLKL lost the necroptotic pathway throughout evolution.

The loss of function of RIPK3 and MLKL in independently evolving lineages (convergent evolution) indicates that gene loss is an important evolutionary mechanism for phenotypic change in these animals and may contribute to similar adaptations. Even though it would be expected that loss of genes is maladaptive, gene loss can be beneficial by providing an evolutionary mechanism for adaptations (50). In fact, if the loss of an existing gene would increase fitness by making a species better adapted to the environment that surrounds it, then gene loss would be an easy solution to an evolutionary problem (50). Necroptosis contributes to innate immunity as a pathogen clearance mechanism (1). However, contrary to apoptosis, in which several highly immunogenic intracellular proteins are sequestered in the dead cell, necroptosis releases DAMPs in the surrounding tissue that promote strong inflammatory responses and result in the attraction of different types of immune cells to the site of infection (51). Studies in mouse models have provided strong evidence that necroptosis is implicated in several inflammatory neurodegenerative diseases, including multiple sclerosis and amyotrophic lateral sclerosis $(52,53)$. Mouse-model experiments identified keratinocyte necroptosis as a trigger of skin inflammation (54) and a correlation between necroptosis and intestinal inflammation has also been established $(55,56)$. Thus, while necroptosis might mediate host defense, its inhibition in certain contexts may lessen disease severity. It is known that excessive inflammation can promote cancer cell growth and metastasis (57). Thus, it is possible that a proinflammatory cell death like necroptosis might promote metastasis and thus, inhibition of this pathway might represent an advantage for regulation of cancer cell growth. Intriguingly, some of the species that are lacking the core necroptotic machinery are known to resist cancer. That is the case for cetaceans, the naked mole-rat and african elephants (58-60). It is also possible that selection against necroptosis in different mammalian lineages could have been driven by different factors depending on the environment or conditions. For example, it was previously suggested that the absence of MLKL in Carnivores reflected a microbe-rich and viruscontaining diet of raw meat, causing evolutionary counterselection against necroptosis (12). Nevertheless, the absence of the necroptotic pathway in independently evolving lineages suggest that the deregulation of this pathway was detrimental for the host organism, which ultimately drove selection against the presence of RIPK3 and MLKL.

As many other viruses, poxviruses express immunomodulatory and host-range factors important for the suppression and evasion of the host innate and adaptive antiviral responses $(61,62)$. VACV protein E3 not only sequester dsRNA through their dsRNA-BD limiting the activation of the innate immune system against the virus infection, but also inhibit the IFN-induced dsRNA dependent protein kinase (PKR), known to be a crucial component of the host innate immunity against viral infection, replication, and spread $(63,64)$. Our results show that the dsRNA$\mathrm{BD}$ of distant E3L proteins present high levels of sequence similarity (Figures 5B, C), which is consistent with the ability of this domain to target conserved pathways present in different hosts. Although the dsRNA sequestration functions of the E3 Cterminal have been clear for decades $(20,65)$, the IFN sensitivity of VACV E3 N-terminal deletion mutants remained unresolved for a long time. Recently, strong evidence showed that the E3 Nterminal domain competes with $\mathrm{ZBP} 1$ to prevent $\mathrm{ZBP} 1$ dependent activation of RIPK3 and consequent necroptosis (38, 66). The model proposed by the authors suggests that during WT$\mathrm{VACV}$ infection, the zNA-BD of E3 binds to VACV-induced Zform nucleic acid and masks it, preventing sensing by ZBP1 and further RIPK3 necroptosis induction $(38,66)$. However, it is interesting that poxviruses like MPXV, MYXV and CePV-TA have E3L homologs that present a complete dsRNA-BD but not zNA-BD (Figure 5B). In VACV-E3 $\Delta 83 \mathrm{~N}$-infected cells (mutant lacking the first 83 aa corresponding to the $\mathrm{zNA}-\mathrm{BD}$ ), the absence of the zNA-BD facilitates ZBP1 to sense VACV-induced PAMPs and initiate necroptosis induction (38). Therefore, it is expected that E3L homologues that lack N-terminal zNA domains, like CePV-TA-21 and M029, cannot prevent Z-form nucleic acid sensing triggering necroptosis induction and early abortion of viral replication. Like CePV-TA-20, F3 protein is also missing several amino acids in the $\mathrm{N}$-terminal region and presents high conservation in areas that are known to bind to zNA (Figure 5C). Nevertheless, F3 protein seems to have lost the ability to compete with ZBP1 and inhibit sensing (unpublished data). It was previously shown that the N-terminus of VACV E3 is necessary for IFN resistance in JC cells since VACV-E3 $\triangle 37 \mathrm{~N}$ (mutant mimicking MPXV E3 zNA-BD) did not initiate DNA replication (67). However, MPXV was able to replicate efficiently in the same cells, despite having a partial N-terminal $\mathrm{zNA}-\mathrm{BD}$, suggesting that the predicted binding to $\mathrm{z}$-form nucleic acid was intragenic and downstream of $\mathrm{z}$-NA sensing, rather than related to the ability of F3 zNA-BD to mask $\mathrm{z}$-form nucleic acid (67).

Interestingly, inactivation of necroptosis in Lagomorphs and Cetaceans seems to correlate with the absence of the E3L zNA$\mathrm{BD}$ in their naturally infecting poxviruses, namely leporipoxviruses (MYXV and SFV) and cetaceanpoxviruses (CePV-TA), respectively. Monkeypox is a viral zoonosis endemic to central and western Africa areas where African rope squirrels and other rodents are likely reservoir hosts (68). Interestingly, the absence of a functional N-terminus in MPXV F3 protein also seems to correlate with the fact that some rodents 
appear incapable of undergoing necroptosis. Like MPXV, leporipoxviruses and CePV-TA pathogenesis are restricted to only certain species and have little or no pathogenesis capability in all others $(41,67,69,70)$. Infection of the same host over hundreds of years or even millennia may drive the evolution of each virus to rapidly evolve to a fitness peak in a given host environment. Previous niche-filling models (71-73) emphasize the role of host interactions in shaping virus evolution. According to these models, as hosts diversify and speciate over longer evolutionary periods, viral host factors that aim to counter the host antiviral functions are subject to continuous changes. Indeed, it is known that genes associated with host antiviral mechanisms present high evolutionary rates and are often under positive selection $(15,74,75)$. Here, we suggest that during the evolution of these poxviruses, the loss of the zNA-BD did not present a disadvantage in the host organism; therefore, this trait was maintained, which reflects how these viruses adapt as their niche changed.

\section{CONCLUSION}

The disruption of necroptosis in independently evolving lineages suggests a convergent evolutionary loss of this pathway, probably reflecting an important selective mechanism for phenotypic change. Interestingly, we also found a strong correlation between the disruption of necroptosis in leporids and cetaceans and the absence of the E3L zNA-BD (responsible for necroptosis inhibition) in their naturally infecting poxviruses as in the case of MYXV and CePV-TA, respectively. Overall, our study provides the first comprehensive picture of the molecular evolution of necroptosis in mammals, highlighting the importance of gene/pathway loss for the process of species adaptation and suggesting that it is a true pathogen-response pathway that is not required for normal mammalian development. Moreover, this study sheds some light on a coevolutionary relationship between poxviruses and their hosts, emphasizing the role of host adaptation in shaping virus evolution.

\section{REFERENCES}

1. Xia X, Lei L, Wang S, Hu J, Zhang G. Necroptosis and its Role in Infectious Diseases. Apoptosis (2020) 25:169-78. doi: 10.1007/s10495-019-01589-X

2. Nogusa S, Thapa RJ, Dillon CP, Liedmann S, Oguin TH3rd, Ingram JP, et al. RIPK3 Activates Parallel Pathways of MLKL-Driven Necroptosis and FADDMediated Apoptosis to Protect Against Influenza A Virus. Cell Host Microbe (2016) 20:13-24. doi: 10.1016/j.chom.2016.05.011

3. Upton JW, Chan FK-M. Staying Alive: Cell Death in Antiviral Immunity. Mol Cell (2014) 54:273-80. doi: 10.1016/j.molcel.2014.01.027

4. Sun L, Wang H, Wang Z, He S, Chen S, Liao D, et al. Mixed Lineage Kinase Domain-Like Protein Mediates Necrosis Signaling Downstream of RIP3 Kinase. Cell (2012) 148:213-27. doi: 10.1016/j.cell.2011.11.031

5. Moerke C, Bleibaum F, Kunzendorf U, Krautwald S. Combined Knockout of RIPK3 and MLKL Reveals Unexpected Outcome in Tissue Injury and Inflammation. Front Cell Dev Biol (2019) 7:19. doi: 10.3389/ fcell.2019.00019

6. He S, Wang L, Miao L, Wang T, Du F, Zhao L, et al. Receptor Interacting Protein Kinase-3 Determines Cellular Necrotic Response to TNF-Alpha. Cell (2009) 137:1100-11. doi: 10.1016/j.cell.2009.05.021

\section{DATA AVAILABILITY STATEMENT}

The datasets presented in this study can be found in online repositories. The names of the repository/repositories and accession number(s) can be found in the article/Supplementary Material.

\section{AUTHOR CONTRIBUTIONS}

AÁ-P, BJ, LC, GM, MR and PE contributed to conception and design of the study. AÁ-P, FN and LA were responsible for the acquisition and statistical analysis of the data. AA-P, LC, LA, BJ and $\mathrm{PE}$ contributed for the interpretation of data. AÁ-P wrote the first draft of manuscript. MR, GM and PE acquired funding to support the study. All authors contributed to the article and approved the submitted version.

\section{FUNDING}

This work was supported by Foundation for Science and Technology (FCT), which supported the doctoral fellowship of AÁ-P (ref.SFRH/ $\mathrm{BD} / 128752 / 2017)$, the investigator grant of PJE (IF/00376/2015) and the project UIDB/50027/2020 (Base). This article is also a result of the project NORTE-01-0145-FEDER-000007, supported by Norte Portugal Regional Operational Programme (NORTE2020), under the PORTUGAL 2020 Partnership Agreement, through the European Regional Development Fund (ERDF). GM and MMR's research is supported by the National Institute of Health (NIH) grants R01AI080607 and R01AI148302.

\section{SUPPLEMENTARY MATERIAL}

The Supplementary Material for this article can be found online at: https://www.frontiersin.org/articles/10.3389/fimmu.2021. 747737/full\#supplementary-material

7. Zhang D-W, Shao J, Lin J, Zhang N, Lu B-J, Lin S-C, et al. RIP3, an Energy Metabolism Regulator That Switches TNF-Induced Cell Death From Apoptosis to Necrosis. Science (2009) 325:332-6. doi: 10.1126/ science. 1172308

8. Upton JW, Kaiser WJ, Mocarski ES. Virus Inhibition of RIP3-Dependent Necrosis. Cell Host Microbe (2010) 7:302-13. doi: 10.1016/j.chom.2010.03.006

9. Upton JW, Kaiser WJ, Mocarski ES. DAI/ZBP1/DLM-1 Complexes With RIP3 to Mediate Virus-Induced Programmed Necrosis That Is Targeted by Murine Cytomegalovirus vIRA. Cell Host Microbe (2012) 11:290-7. doi: 10.1016/j.chom.2012.01.016

10. He S, Liang Y, Shao F, Wang X. Toll-Like Receptors Activate Programmed Necrosis in Macrophages Through a Receptor-Interacting Kinase-3-Mediated Pathway. Proc Natl Acad Sci USA (2011) 108:20054-9. doi: 10.1073/pnas.1116302108

11. Kaiser WJ, Upton JW, Mocarski ES. Viral Modulation of Programmed Necrosis. Curr Opin Virol (2013) 3:296-306. doi: 10.1016/j.coviro.2013.05.019

12. Dondelinger Y, Hulpiau P, Saeys Y, Bertrand MJM, Vandenabeele P. An Evolutionary Perspective on the Necroptotic Pathway. Trends Cell Biol (2016) 26:721-32. doi: 10.1016/j.tcb.2016.06.004

13. Sharma V, Hecker N, Roscito JG, Foerster L, Langer BE, Hiller M. A Genomics Approach Reveals Insights Into the Importance of Gene Losses 
for Mammalian Adaptations. Nat Commun (2018) 9:1215. doi: 10.1038/ s41467-018-03667-1

14. Águeda-Pinto A, Castro LFC, Esteves PJ. The Evolution of S100A7: An Unusual Gene Expansion in Myotis Bats. BMC Evol Biol (2019) 19:102. doi: 10.1186/s12862-019-1433-0

15. Águeda-Pinto A, Lemos de Matos A, Pinheiro A, Neves F, de Sousa-Pereira P, Esteves PJ. Not So Unique to Primates: The Independent Adaptive Evolution of TRIM5 in Lagomorpha Lineage. PloS One (2019) 14:e0226202. doi: 10.1371/journal.pone.0226202

16. de Matos AL, van der Loo W, Areal H, Lanning DK, Esteves PJ. Study of Sylvilagus Rabbit TRIM5 $\alpha$ Species-Specific Domain: How Ancient Endoviruses Could Have Shaped the Antiviral Repertoire in Lagomorpha. BMC Evol Biol (2011) 11:294. doi: 10.1186/1471-2148-11-294

17. Alves LQ, Ruivo R, Fonseca MM, Lopes-Marques M, Ribeiro P, Castro LFC. PseudoChecker: An Integrated Online Platform for Gene Inactivation Inference. Nucleic Acids Res (2020) 48:W321-31. doi: 10.1093/nar/gkaa408

18. Ranwez V, Douzery EJP, Cambon C, Chantret N, Delsuc F. MACSE V2: Toolkit for the Alignment of Coding Sequences Accounting for Frameshifts and Stop Codons. Mol Biol Evol (2018) 35:2582-4. doi: 10.1093/molbev/ msy159

19. Kearse M, Moir R, Wilson A, Stones-Havas S, Cheung M, Sturrock S, et al. Geneious Basic: An Integrated and Extendable Desktop Software Platform for the Organization and Analysis of Sequence Data. Bioinformatics (2012) 28:1647-9. doi: 10.1093/bioinformatics/bts199

20. Thompson JD, Higgins DG, Gibson TJ. CLUSTAL W: Improving the Sensitivity of Progressive Multiple Sequence Alignment Through Sequence Weighting, Position-Specific Gap Penalties and Weight Matrix Choice. Nucleic Acids Res (1994) 22:4673-80. doi: 10.1093/nar/22.22.4673

21. Kumar S, Stecher G, Li M, Knyaz C, Tamura K. MEGA X: Molecular Evolutionary Genetics Analysis Across Computing Platforms. Mol Biol Evol (2018) 35:1547-9. doi: 10.1093/molbev/msy096

22. Darriba D, Taboada GL, Doallo R, Posada D. ProtTest 3: Fast Selection of Best-Fit Models of Protein Evolution. Bioinformatics (2011) 27:1164-5. doi: 10.1093/bioinformatics/btr088

23. Pond SLK, Frost SDW. Datamonkey: Rapid Detection of Selective Pressure on Individual Sites of Codon Alignments. Bioinformatics (2005) 21:2531-3. doi: 10.1093/bioinformatics/bti320

24. Kosakovsky Pond SL, Frost SDW. Not So Different After All: A Comparison of Methods for Detecting Amino Acid Sites Under Selection. Mol Biol Evol (2005) 22:1208-22. doi: 10.1093/molbev/msi105

25. Murrell B, Wertheim JO, Moola S, Weighill T, Scheffler K, Kosakovsky Pond SL. Detecting Individual Sites Subject to Episodic Diversifying Selection. PloS Genet (2012) 8:e1002764. doi: 10.1371/journal.pgen.1002764

26. Murrell B, Moola S, Mabona A, Weighill T, Sheward D, Kosakovsky Pond SL, et al. FUBAR: A Fast, Unconstrained Bayesian Approximation for Inferring Selection. Mol Biol Evol (2013) 30:1196-205. doi: 10.1093/molbev/mst030

27. He S, Wang X. RIP Kinases as Modulators of Inflammation and Immunity. Nat Immunol (2018) 19:912-22. doi: 10.1038/s41590-018-0188-x

28. Kosiol C, Vinar T, da Fonseca RR, Hubisz MJ, Bustamante CD, Nielsen R, et al. Patterns of Positive Selection in Six Mammalian Genomes. PloS Genet (2008) 4:e1000144. doi: 10.1371/journal.pgen.1000144

29. Mifflin L, Ofengeim D, Yuan J. Receptor-Interacting Protein Kinase 1 (RIPK1) as a Therapeutic Target. Nat Rev Drug Discovery (2020) 19:553-71. doi: 10.1038/s41573-020-0071-y

30. Sun X, Yin J, Starovasnik MA, Fairbrother WJ, Dixit VM. Identification of a Novel Homotypic Interaction Motif Required for the Phosphorylation of Receptor-Interacting Protein (RIP) by RIP3. J Biol Chem (2002) 277:9505-11. doi: $10.1074 /$ jbc.M109488200

31. Li J, McQuade T, Siemer AB, Napetschnig J, Moriwaki K, Hsiao Y-S, et al. The RIP1/RIP3 Necrosome Forms a Functional Amyloid Signaling Complex Required for Programmed Necrosis. Cell (2012) 150:339-50. doi: 10.1016/ j.cell.2012.06.019

32. Meng H, Liu Z, Li X, Wang H, Jin T, Wu G, et al. Death-Domain Dimerization-Mediated Activation of RIPK1 Controls Necroptosis and RIPK1-Dependent Apoptosis. Proc Natl Acad Sci USA (2018) 115:E2001-9. doi: 10.1073/pnas.1722013115

33. Melo-Ferreira J, Lemos de Matos A, Areal H, Lissovsky AA, Carneiro M, Esteves PJ. The Phylogeny of Pikas (Ochotona) Inferred From a Multilocus
Coalescent Approach. Mol Phylogenet Evol (2015) 84:240-4. doi: 10.1016/ j.ympev.2015.01.004

34. Ge D, Wen Z, Xia L, Zhang Z, Erbajeva M, Huang C, et al. Evolutionary History of Lagomorphs in Response to Global Environmental Change. PloS One (2013) 8:e59668. doi: 10.1371/journal.pone.0059668

35. McGowen MR, Tsagkogeorga G, Álvarez-Carretero S, Dos Reis M, Struebig M, Deaville R, et al. Phylogenomic Resolution of the Cetacean Tree of Life Using Target Sequence Capture. Syst Biol (2020) 69:479-501. doi: 10.1093/ sysbio/syz068

36. Huelsmann M, Hecker N, Springer MS, Gatesy J, Sharma V, Hiller M. Genes Lost During the Transition From Land to Water in Cetaceans Highlight Genomic Changes Associated With Aquatic Adaptations. Sci Adv (2019) 5: eaaw6671. doi: 10.1126/sciadv.aaw6671

37. Kawasaki K, Mikami M, Goto M, Shindo J, Amano M, Ishiyama M. The Evolution of Unusually Small Amelogenin Genes in Cetaceans; Pseudogenization, X-Y Gene Conversion, and Feeding Strategy. J Mol Evol (2020) 88:122-35. doi: 10.1007/s00239-019-09917-0

38. Koehler H, Cotsmire S, Langland J, Kibler KV, Kalman D, Upton JW, et al. Inhibition of DAI-Dependent Necroptosis by the Z-DNA Binding Domain of the Vaccinia Virus Innate Immune Evasion Protein, E3. Proc Natl Acad Sci USA (2017) 114:11506-11. doi: 10.1073/pnas.1700999114

39. Kim Y-G, Lowenhaupt K, Oh D-B, Kim KK, Rich A. Evidence That Vaccinia Virulence Factor E3L Binds to Z-DNA In Vivo: Implications for Development of a Therapy for Poxvirus Infection. Proc Natl Acad Sci USA (2004) 101:1514-8. doi: 10.1073/pnas.0308260100

40. Kim Y-G, Muralinath M, Brandt T, Pearcy M, Hauns K, Lowenhaupt K, et al. A Role for Z-DNA Binding in Vaccinia Virus Pathogenesis. Proc Natl Acad Sci USA (2003) 100:6974-9. doi: 10.1073/pnas.0431131100

41. Rodrigues TCS, Subramaniam K, Varsani A, McFadden G, Schaefer AM, Bossart GD, et al. Genome Characterization of Cetaceanpox Virus From a Managed Indo-Pacific Bottlenose Dolphin (Tursiops Aduncus). Virus Res (2020) 278:197861. doi: 10.1016/j.virusres.2020.197861

42. Rahman MM, McFadden G. Myxoma Virus-Encoded Host Range Protein M029: A Multifunctional Antagonist Targeting Multiple Host Antiviral and Innate Immune Pathways. Vaccines (Basel) (2020) 8:2-3. doi: 10.3390/ vaccines8020244

43. Nailwal H, Chan FK-M. Necroptosis in Anti-Viral Inflammation. Cell Death Differ (2019) 26:4-13. doi: 10.1038/s41418-018-0172-x

44. Orzalli MH, Kagan JC. Apoptosis and Necroptosis as Host Defense Strategies to Prevent Viral Infection. Trends Cell Biol (2017) 27:800-9. doi: 10.1016/ j.tcb.2017.05.007

45. Dannappel M, Vlantis K, Kumari S, Polykratis A, Kim C, Wachsmuth L, et al. RIPK1 Maintains Epithelial Homeostasis by Inhibiting Apoptosis and Necroptosis. Nature (2014) 513:90-4. doi: 10.1038/nature13608

46. Rickard JA, Anderton H, Etemadi N, Nachbur U, Darding M, Peltzer N, et al. TNFR1-Dependent Cell Death Drives Inflammation in Sharpin-Deficient Mice. Elife (2014) 3:7-8. doi: 10.7554/eLife.03464

47. Zhang T, Zhang Y, Cui M, Jin L, Wang Y, Lv F, et al. CaMKII is a RIP3 Substrate Mediating Ischemia- and Oxidative Stress-Induced Myocardial Necroptosis. Nat Med (2016) 22:175-82. doi: 10.1038/nm.4017

48. Günther C, He G-W, Kremer AE, Murphy JM, Petrie EJ, Amann K, et al. The Pseudokinase MLKL Mediates Programmed Hepatocellular Necrosis Independently of RIPK3 During Hepatitis. J Clin Invest (2016) 126:4346-60. doi: 10.1172/JCI87545

49. Dovey CM, Diep J, Clarke BP, Hal AT, McNamara DE, Guo H, et al. MLKL Requires the Inositol Phosphate Code to Execute Necroptosis. Mol Cell (2018) 70:936-48.e7. doi: 10.1016/j.molcel.2018.05.010

50. Albalat R, Cañestro C. Evolution by Gene Loss. Nat Rev Genet (2016) 17:37991. doi: 10.1038/nrg.2016.39

51. Kaczmarek A, Vandenabeele P, Krysko DV. Necroptosis: The Release of Damage-Associated Molecular Patterns and Its Physiological Relevance. Immunity (2013) 38:209-23. doi: 10.1016/j.immuni.2013.02.003

52. Ito Y, Ofengeim D, Najafov A, Das S, Saberi S, Li Y, et al. RIPK1 Mediates Axonal Degeneration by Promoting Inflammation and Necroptosis in ALS. Science (2016) 353:603-8. doi: 10.1126/science.aaf6803

53. Ofengeim D, Ito Y, Najafov A, Zhang Y, Shan B, DeWitt JP, et al. Activation of Necroptosis in Multiple Sclerosis. Cell Rep (2015) 10:1836-49. doi: 10.1016/ j.celrep.2015.02.051 
54. Bonnet MC, Preukschat D, Welz P-S, van Loo G, Ermolaeva MA, Bloch W, et al. The Adaptor Protein FADD Protects Epidermal Keratinocytes From Necroptosis In Vivo and Prevents Skin Inflammation. Immunity (2011) 35:572-82. doi: 10.1016/j.immuni.2011.08.014

55. Welz P-S, Wullaert A, Vlantis K, Kondylis V, Fernández-Majada V, Ermolaeva M, et al. FADD Prevents RIP3-Mediated Epithelial Cell Necrosis and Chronic Intestinal Inflammation. Nature (2011) 477:330-4. doi: 10.1038/ nature 10273

56. Pierdomenico M, Negroni A, Stronati L, Vitali R, Prete E, Bertin J, et al. Necroptosis Is Active in Children With Inflammatory Bowel Disease and Contributes to Heighten Intestinal Inflammation. Am J Gastroenterol (2014) 109:279-87. doi: 10.1038/ajg.2013.403

57. Najafov A, Chen H, Yuan J. Necroptosis and Cancer. Trends Cancer Res (2017) 3:294-301. doi: 10.1016/j.trecan.2017.03.002

58. Tejada-Martinez D, de Magalhães JP, Opazo JC. Positive Selection and Gene Duplications in Tumour Suppressor Genes Reveal Clues About How Cetaceans Resist Cancer. Proc Biol Sci (2021) 288:20202592. doi: 10.1098/ rspb.2020.2592

59. Liang S, Mele J, Wu Y, Buffenstein R, Hornsby PJ. Resistance to Experimental Tumorigenesis in Cells of a Long-Lived Mammal, the Naked Mole-Rat (Heterocephalus Glaber). Aging Cell (2010) 9:626-35. doi: 10.1111/j.14749726.2010.00588.x

60. Abegglen LM, Caulin AF, Chan A, Lee K, Robinson R, Campbell MS, et al. Potential Mechanisms for Cancer Resistance in Elephants and Comparative Cellular Response to DNA Damage in Humans. JAMA (2015) 314:1850-60. doi: 10.1001/jama.2015.13134

61. Oliveira GP, Rodrigues RAL, Lima MT, Drumond BP, Abrahão JS. Poxvirus Host Range Genes and Virus-Host Spectrum: A Critical Review. Viruses (2017) 9:9-12. doi: 10.3390/v9110331

62. Werden SJ, Rahman MM, McFadden G. Poxvirus Host Range Genes. Adv Virus Res (2008) 71:135-71. doi: 10.1016/S0065-3527(08)00003-1

63. Davies MV, Chang HW, Jacobs BL, Kaufman RJ. The E3L and K3L Vaccinia Virus Gene Products Stimulate Translation Through Inhibition of the Double-Stranded RNA-Dependent Protein Kinase by Different Mechanisms. J Virol (1993) 67:1688-92. doi: 10.1128/jvi.67.3.1688-1692.1993

64. Sharp TV, Moonan F, Romashko A, Joshi B, Barber GN, Jagus R. The Vaccinia Virus E3L Gene Product Interacts With Both the Regulatory and the Substrate Binding Regions of PKR: Implications for PKR Autoregulation. Virology (1998) 250:302-15. doi: 10.1006/viro.1998.9365

65. Chang HW, Jacobs BL. Identification of a Conserved Motif That is Necessary for Binding of the Vaccinia Virus E3L Gene Products to Double-Stranded RNA. Virology (1993) 194:537-47. doi: 10.1006/viro.1993.1292

66. Koehler HS, Cotsmire S, Zhang T, Balachandran S, Upton JW, Langland J, et al. Competition Between E3 and ZBP1 for Z-RNA Dictates Susceptibility to Vaccinia Virus-Induced Necroptosis. SSRN Electron J (2020) 114(43):1150611. doi: $10.2139 / \mathrm{ssrn} .3717768$
67. Arndt WD, Cotsmire S, Trainor K, Harrington H, Hauns K, Kibler KV, et al. Evasion of the Innate Immune Type I Interferon System by Monkeypox Virus. J Virol (2015) 89:10489-99. doi: 10.1128/JVI.00304-15

68. Essbauer S, Pfeffer M, Meyer H. Zoonotic Poxviruses. Vet Microbiol (2010) 140:229-36. doi: 10.1016/j.vetmic.2009.08.026

69. Rahman MM, Liu J, Chan WM, Rothenburg S, McFadden G. Myxoma Virus Protein M029 is a Dual Function Immunomodulator That Inhibits PKR and Also Conscripts RHA/DHX9 to Promote Expanded Host Tropism and Viral Replication. PloS Pathog (2013) 9:e1003465. doi: 10.1371/journal.ppat. 1003465

70. Águeda-Pinto A, Lemos de Matos A, Abrantes M, Kraberger S, Risalde MA, Gortázar C, et al. Genetic Characterization of a Recombinant Myxoma Virus in the Iberian Hare (). Viruses (2019) 11:3-13. doi: 10.3390/v11060530

71. Cooper N, Jetz W, Freckleton RP. Phylogenetic Comparative Approaches for Studying Niche Conservatism. J Evol Biol (2010) 23:2529-39. doi: 10.1111/ j.1420-9101.2010.02144.x

72. Holt RD. Bringing the Hutchinsonian Niche Into the 21st Century: Ecological and Evolutionary Perspectives. Proc Natl Acad Sci USA (2009) 106 Suppl 2:19659-65. doi: 10.1073/pnas.0905137106

73. Simmonds P, Aiewsakun P, Katzourakis A. Prisoners of War - Host Adaptation and Its Constraints on Virus Evolution. Nat Rev Microbiol (2019) 17:321-8. doi: 10.1038/s41579-018-0120-2

74. van der Lee R, Wiel L, van Dam TJP, Huynen MA. Genome-Scale Detection of Positive Selection in Nine Primates Predicts Human-Virus Evolutionary Conflicts. Nucleic Acids Res (2017) 45:10634-48. doi: 10.1093/nar/gkx704

75. Münk C, Willemsen A, Bravo IG. An Ancient History of Gene Duplications, Fusions and Losses in the Evolution of APOBEC3 Mutators in Mammals. BMC Evol Biol (2012) 12:71. doi: 10.1186/1471-2148-12-71

Conflict of Interest: The authors declare that the research was conducted in the absence of any commercial or financial relationships that could be construed as a potential conflict of interest.

Publisher's Note: All claims expressed in this article are solely those of the authors and do not necessarily represent those of their affiliated organizations, or those of the publisher, the editors and the reviewers. Any product that may be evaluated in this article, or claim that may be made by its manufacturer, is not guaranteed or endorsed by the publisher.

Copyright (๑ 2021 Águeda-Pinto, Alves, Neves, McFadden, Jacobs, Castro, Rahman and Esteves. This is an open-access article distributed under the terms of the Creative Commons Attribution License (CC BY). The use, distribution or reproduction in other forums is permitted, provided the original author(s) and the copyright owner(s) are credited and that the original publication in this journal is cited, in accordance with accepted academic practice. No use, distribution or reproduction is permitted which does not comply with these terms. 